\title{
Numerical Solution for Eigenvalues and Eigenfunctions of a Hermitian Kernel and an Error Estimate
}

\author{
By E. Rakotch
}

\begin{abstract}
New error estimates for eigenvalues of symmetric integral equations are obtained. These estimates are applicable to a more general class of integration methods and, in many cases, are better than those of Wielandt. For every eigenvalue, a numerical solution for the corresponding eigenfunction is also obtained. Whenever the exact eigenvalue happens to be simple, an error estimate for the corresponding eigenfunction is also derived.
\end{abstract}

1. Introduction. Let $K(x, t)$ be a Hermitian kernel defined in $I \times I$, where $I \equiv$ $[a, b]$, i.e., $K(t, x)=\overline{K(x, t)}$, such that

$$
F(x) \equiv \int_{a}^{b}|K(x, t)|^{2} d t \quad \text { is bounded in } I ;
$$

then all the characteristic values $\mu_{i}$ of $K(x, t)$ are real and there exists an orthonormal set $\left\{y_{i}(x)\right\}$ of characteristic functions [5], i.e.,

$$
\int_{a}^{b} K(x, t) y_{i}(t) d t=\mu_{i} y_{i}(x), \quad\left(y_{i}, y_{j}\right)=\delta_{i j},
$$

where $(u, v) \equiv \int_{a}^{b} u(x) \overline{v(x)} d x$ is the scalar product of two complex functions $u(x)$, $v(x) \in L_{2}(I) \equiv\{z(x) \mid(z, z)<\infty\}$.

Further, let $S$ be a rule of numerical integration with weights $w_{i n}>0$ and nodes $x_{i n} \in I, i=1, \ldots, n$, by which the approximation $\int_{a}^{b} f(x) d x \approx \sum_{i=1}^{n} w_{i n} f\left(x_{i n}\right)$ is made.

To obtain a numerical solution for the characteristic values of $K(x, t)$, Wielandt [9] replaced the original problem by the sequence of eigenproblems

$$
K^{(n)} y_{i}^{(n)}=\mu_{i n} y_{i}^{(n)}, \quad K_{i j}^{(n)} \equiv w_{j n} K\left(x_{i n}, x_{j n}\right), \quad i, j=1, \ldots, n,
$$

with real $\mu_{i n}$ and $n$ linearly independent eigenvectors $y_{i}^{(n)}$, for a class of integration rules possessing the properties

$$
\begin{aligned}
\lim _{n \rightarrow \infty} \sum_{i=1}^{n} w_{i n} f\left(x_{i n}\right) & =\int_{a}^{b} f(x) d x \quad \text { for every } f(x) \in C(I), \\
\sum_{i=1}^{n} w_{i n} & =b-a ;
\end{aligned}
$$

the eigenvalues $\mu_{k n}, k=1, \ldots, n$, are then taken by Wielandt as approximations, which also converge as $n \rightarrow \infty$, to the corresponding characteristic values of $K(x, t)$. To specify this correspondence, the following assumptions are made:

Received July 2, 1972; revised December 2, 1974.

AMS (MOS) subject classifications (1970). Primary 65 R05.

Copyright $\odot$ 1975, American Mathematical Society 
Let $V \equiv\left\{\alpha_{1}, \ldots, \alpha_{m}\right\}$ be a subset of the set $R$ of all eigenvalues of a square matrix $A$ or of all characteristic values of a kernel $F(x, t)$ defined in $I \times I$, and let $W \equiv$ $\left\{z^{2} \mid z \in V\right\}$; then

(a) if $\alpha_{1}, \ldots, \alpha_{m}$ are the $m$ largest (smallest) real elements of $R$ such that $\alpha_{1} \geqslant \alpha_{2} \geqslant \ldots \geqslant \alpha_{m}\left(\alpha_{1} \leqslant \alpha_{2} \leqslant \ldots \leqslant \alpha_{m}\right)$, then every $\alpha_{i} \neq \alpha_{m}$ with multiplicity $r_{i}>1$ occurs $r_{i}$ times in $V$,

(b) if $\alpha_{1}, \ldots, \alpha_{m}$ are the $m$ real elements of $R$ the largest modulus such that $\left|\alpha_{1}\right| \geqslant\left|\alpha_{2}\right| \geqslant \ldots \geqslant\left|\alpha_{m}\right|$ and there are $r_{i}$ real elements of $R$ of modulus $\left|\alpha_{i}\right|$, then every $\alpha_{i}^{2} \neq \alpha_{m}^{2}$ occurs $r_{i}$ times in $W$.

The problem which arises now is what is the best error estimate for the eigenvalues $\mu_{k n}$ of (2). In this context, and with the above assumptions, Wielandt obtained for those integration rules, which we shall call convergent with respect to $K(x, t)$-i.e. the sequence

$$
\eta_{n}(x, t) \equiv \sum_{i=1}^{n} w_{i n} K\left(x, x_{i n}\right) K\left(x_{i n}, t\right)-\int_{a}^{b} K(x, z) K(z, t) d z
$$

of the error functions for $f(z) \equiv K(x, z) K(z, t)$ converges to 0 uniformly in $I \times I$, the following result:

Let $\mu_{1 n}^{+} \geqslant \mu_{2 n}^{+} \geqslant \ldots \geqslant \mu_{r n}^{+}>0>\mu_{s n}^{-} \geqslant \ldots \geqslant \mu_{2 n}^{-} \geqslant \mu_{1 n}^{-}$be the $r$ largest positive and the $s$ smallest negative eigenvalues of (2), and let

$$
\mu_{1}^{+} \geqslant \mu_{2}^{+} \geqslant \ldots \geqslant \mu_{r}^{+}>0>\mu_{s}^{-} \geqslant \ldots \geqslant \mu_{2}^{-} \geqslant \mu_{1}^{-}
$$

be the corresponding characteristic values of $K(x, t)$; then

$$
\mu_{i}^{+}=\lim _{n \rightarrow \infty} \mu_{i n}^{+}, \quad \mu_{j}^{-}=\lim _{n \rightarrow \infty} \mu_{j n}^{-}, \quad i=1, \ldots, r, j=1, \ldots, s,
$$

and this convergence is uniform in $i$ and $j$, i.e.,

$$
\sigma_{k n} \equiv\left|\mu_{k n}-\mu_{k}\right| \leqslant q_{n}, \quad \lim _{n \rightarrow \infty} q_{n}=0,
$$

where either

$$
\mu_{k n}=\mu_{k n}^{+}, \quad \mu_{k}=\mu_{k}^{+} \text {, or } \mu_{k n}=\mu_{k n}^{-}, \quad \mu_{k}=\mu_{k}^{-} \text {. }
$$

Baker [2] obtained convergence properties of a similar type for simple characteristic values of $K(x, t)$. The best estimate obtained by Wielandt is $q_{n}=O\left(\sqrt{\epsilon_{n}}\right)$, where $\epsilon_{n} \equiv \max _{I \times I}\left|\eta_{n}(x, t)\right|$ and $\eta_{n}(x, t)$ is defined by (4), whereas that of Baker is $q_{n}=$ $O\left(\max w_{i n}\right)$. Other authors ([1], [3]) obtained better bounds, but only for the distance of every eigenvalue $\mu_{k n}$ to the nearest characteristic value of $K(x, t)$. In this paper improved estimates of the form (see Theorem 1 at the beginning of Section 4)

$$
\sigma_{k n}=\left[\max \left(\left|\mu_{k n}\right|,\left|\mu_{k}\right|\right)\right]^{-1} \rho_{n}, \quad \rho_{n}=O\left(\epsilon_{n}\right),
$$

are obtained, which generalizes Wielandt's convergence theorems for all integration rules which are convergent with respect to $K(x, t)$ and satisfy (3). Moreover, the new result enables application of integration rules, which are convergent with respect to $K(x, t)$, to kernels which exhibit singular behavior in $I \times I$ and for which, therefore, no solution can be found within the scope of Wielandt's and Baker's papers (see Example 2 in Sec- 
tion 2). As a consequence, an error estimate for the numerical solution of (1), convergent to 0 uniformly in $I$ for every integration rule which is convergent with respect to $K(x, t)$, is derived. The new error estimates for eigenvalues are to be interpreted as follows: our estimates are better than those of Wielandt for the first $m_{n}$ eigenvalues $\mu_{k n}$ such that $\max \left(\left|\mu_{k n}\right|,\left|\mu_{k}\right|\right)>C \sqrt{\epsilon_{n}}$ for some $C>0$, where the sequence $m_{n}$ tends to infinity; for other eigenvalues both our and Wielandt's estimates are of the same order of magnitude, namely $O\left(\sqrt{\epsilon_{n}}\right)$, and ours are not necessarily better.

2. Numerical Results. To illustrate the superiority of the new error estimates given by Theorem 1 in Section 4, two numerical examples are presented. To the second of our examples Wielandt's method does not apply.

In the tables of results given below, $\mu_{l n}^{+}$and $\mu_{l n}^{-}$are the eigenvalues defined in Theorem 2 near the end of Section 4, whereas $y_{l n}^{+}(x)$ and $y_{l n}^{-}(x)$ are the numerical solutions for characteristic functions corresponding to $\mu_{l n}^{+}$and $\mu_{l n}^{-}$, respectively, obtained by the procedure described at the end of Section 4. The improved error estimates are those described in Section 5. The error estimates for $y_{l n}^{+}(x)$ and $y_{l n}^{-}(x)$ are those obtained by application of the remark concluding the discussion of Theorem 3 in Section 4.

Example 1. The integral equation is

$$
\int_{0}^{1} \max (x, t) y(t) d t=\mu y(x) .
$$

Characteristic values and characteristic functions are, respectively:

$$
\begin{aligned}
& R^{2} \text { and } \frac{\sqrt{2} \cosh R x}{\cosh R}, \quad \text { where } R \text { is the positive root of the equation } z \tanh z=1 \\
& -r_{N}^{2} \text { and } \frac{\sqrt{2} \cos r_{N} x}{\cos r_{N}}, \quad \begin{array}{l}
N=1,2, \ldots, \text { where } 0<r_{1}<r_{2}<\ldots \text { are the positive } \\
\text { roots of the equation } z \tan z+1=0 .
\end{array}
\end{aligned}
$$

The integration rule $S$ mentioned at the introduction is the trapezoidal rule.

To obtain $\alpha_{n}, \beta_{n}$ and $\gamma_{n}$, as defined in (24), put

$$
\begin{aligned}
& A_{n}(z) \equiv(n-1) z-[(n-1) z], \quad B_{n}(z) \equiv 1-A_{n}(z), \quad C_{n}(z) \equiv A_{n}(z) B_{n}(z), \\
& D_{n}(z) \equiv A_{n}(z)-B_{n}(z), \quad h \equiv(n-1)^{-1}, \quad F_{n}(z) \equiv 1-z-C_{n}(z)\left[3 z-h D_{n}(z)\right] ;
\end{aligned}
$$

then

$$
\eta_{n}(x, t)=\frac{h^{2}}{6} \begin{cases}3 t C_{n}(x)+F_{n}(t), & x \leqslant t \\ 3 x C_{n}(t)+F_{n}(x), & x \geqslant t,\end{cases}
$$

which after a simple, but lengthy, calculation yields

$$
\alpha_{n}^{2}=\frac{h^{4}}{18} \sum_{k=1}^{n-1} \int_{(k-1) h}^{k h} t\left\{t G_{n}(t)+h C_{n}(t) D_{n}(t)\left[G_{n}(t)+0.3 t C_{n}(t)\right]+F_{n}^{2}(t)\right\} d t,
$$

where $G_{n}(t) \equiv 0.3 t+F_{n}(t)$, and

$$
\int_{0}^{1} \eta_{n}^{2}\left(x, x_{i n}\right) d x=\int_{0}^{x_{i n}} \eta_{n}^{2}\left(x, x_{i n}\right) d x+\int_{x_{i n}}^{1} \eta_{n}^{2}\left(x, x_{i n}\right) d x
$$


Each of the above summands is evaluated by the closed Newton-Cotes formula with 7 points. The remark at the end of Section 4 is applied with $p=L=1$.

For comparison with Wielandt's results the error estimates for the negative eigenvalues $\mu_{l n}^{-}$, together with error estimates for the numerical solutions for characteristic functions, are presented in the following table:

TABLE 1

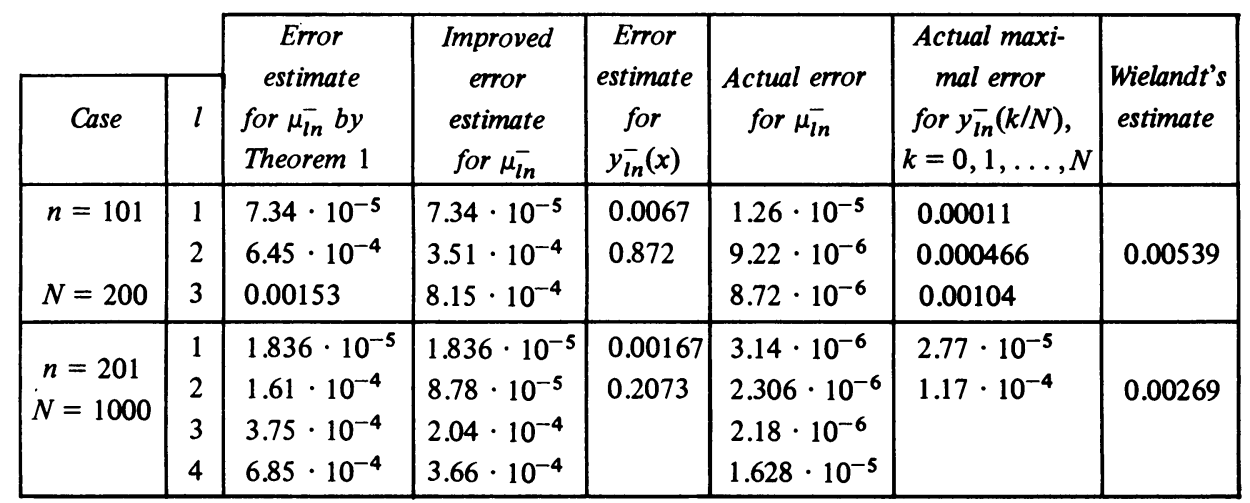

It is to be noted that as the initial error estimates for the eigenvalues $\overline{\mu_{l n}}$ tend to grow with $l$, they are better than those of Wielandt only for some first eigenvalues. To obtain a comparable error estimate unobtainable by Theorem 1 for other eigenvalues, the bound $q_{n}$ defined by (26) with the optimal $C=\sqrt{0.5(1+\sqrt{5})}$ can be taken.

Example 2. The integral equation is

$$
\int_{0}^{1}(1+i \sqrt{x}-i \sqrt{t})^{-1} y(t) d t=\mu y(x) .
$$

The exact solution is unknown.

The integration rule, which is derived by the transformation $u=z^{2}$ for the integral $\int_{0}^{1} K(x, z) K(z, t) d z$ and application of the Gauss quadrature with weights $\omega_{\text {in }}$ and nodes $\xi_{\text {in }}, i=1, \ldots, n$, is defined by

$$
w_{\text {in }} \equiv 2 \omega_{\text {in }} \xi_{\text {in }}, \quad x_{\text {in }} \equiv \xi_{\text {in }}^{2}, \quad i=1, \ldots, n ;
$$

therefore, using our definition (4) $[7$, p. 48],

$$
\begin{aligned}
\eta_{n}(x, t) & =2 c_{n}\left[\frac{\partial^{2 n}}{\partial u^{2 n}} u K\left(x, u^{2}\right) K\left(u^{2}, t\right)\right]_{u=\xi} \\
& =\frac{2(2 n) ! c_{n}}{\sqrt{x}-\sqrt{t}-2 i}\left[\frac{\sqrt{x}-i}{(\xi-\sqrt{x}+i)^{2 n+1}}-\frac{\sqrt{t}+i}{(\xi-\sqrt{t}-i)^{2 n+1}}\right],
\end{aligned}
$$

where $c_{n}=\left[\left(\begin{array}{c}2 n \\ n\end{array}\right)^{2}(2 n+1) !\right]^{-1}$ and $0<\xi=\xi(x, t)<1$, and consequently

$$
\left|\eta_{n}(x, t)\right| \leqslant\left(\begin{array}{c}
2 n \\
n
\end{array}\right)^{-2}(2 n+1)^{-1}(\sqrt{1+x}+\sqrt{1+t}) .
$$

The error estimates, with those for $y_{l n}^{+}(x)$ obtained by application of the remark at the end of Section 4 with $p=1 / 2$ and $L=1$, are given in the table below: 
TABLE 2

\begin{tabular}{|c|c|c|c|c|}
\cline { 2 - 5 }$n$ & $l$ & $\begin{array}{c}\text { Error estimate for } \mu_{\text {ln }}^{+} \\
\text {by Theorem } 1\end{array}$ & $\begin{array}{c}\text { Improved error } \\
\text { estimate for } \mu_{l n}^{+}\end{array}$ & $\begin{array}{c}\text { Error estimate } \\
\text { for } y_{l n}^{+}(x)\end{array}$ \\
\hline \multirow{6}{*}{6} & 1 & $2.31 \cdot 10^{-7}$ & $2.31 \cdot 10^{-7}$ & $8.8 \cdot 10^{-7}$ \\
& 2 & $1.015 \cdot 10^{-5}$ & $5.08 \cdot 10^{-6}$ & 0.00383 \\
& 3 & $2.12 \cdot 10^{-4}$ & $1.033 \cdot 10^{-4}$ & \\
\hline & 1 & $9.1 \cdot 10^{-10}$ & $9.1 \cdot 10^{-10}$ & $3.41 \cdot 10^{-9}$ \\
8 & 2 & $4 \cdot 10^{-8}$ & $2 \cdot 10^{-8}$ & $1.47 \cdot 10^{-5}$ \\
& 3 & $8.15 \cdot 10^{-7}$ & $4.08 \cdot 10^{-7}$ & 0.11 \\
\hline
\end{tabular}

The approximations for $n=9$ rounded to 10 digits are:

$$
\mu_{1 n}^{+} \approx 0.9543482459, \mu_{2 n}^{+} \approx 0.0434068611, \quad \mu_{3 n}^{+} \approx 0.0021321407 \text {. }
$$

3. Numerical Solution for a Characteristic Function. Since the new results, presented in Section 2, refer also to an error estimate for the corresponding characteristic function, an appropriate definition of the approximate solution for a characteristic function, which converges to the corresponding characteristic function, is to be given. To obtain such a definition, observe first that by the similarity relation between the matrix $K^{(n)}$ of (2) and the Hermitian matrix $H$ with $H_{i j} \equiv K\left(x_{i n}, x_{j n}\right) \sqrt{w_{i n} w_{j n}}, i, j=$ $1, \ldots, n$, the eigenvector $y_{k}^{(n)}$ is related to the corresponding eigenvector $z_{k}$ of $H$ by $z_{k i}=y_{k i}^{(n)} \sqrt{w_{i n}}, i=1, \ldots, n$. Further, define a new scalar product $(u, v)_{n}$ of two vectors $u, v$ in $C_{n}$-the $n$-dimensional complex Euclidean space-and a new norm $|u|_{n}$ in $C_{n}$, by

$$
(u, v)_{n} \equiv \sum_{i=1}^{n} w_{i n} u_{i} \bar{v}_{i}, \quad|u|_{n} \equiv \sqrt{(u, u)_{n}},
$$

and denote by $\|f\| \equiv \sqrt{(f, f)}$ the norm of a complex function $f(x)$; therefore, if the eigenvectors $z_{k}, k=1, \ldots, n$, of $H$ are chosen so as to form an orthonormal set, then

$$
\left(y_{p}^{(n)}, y_{q}^{(n)}\right)_{n}=\delta_{p q}, \quad p, q=1, \ldots, n
$$

For every eigenvector $y_{k}^{(n)}$ of (2) with $\mu_{k n} \neq 0$, define now the numerical solution $y_{k n}(x)$ for a characteristic function generated by $y_{k}^{(n)}$, which also satisfies $y_{k n}\left(x_{i n}\right)=$ $y_{k i}^{(n)}, i=1, \ldots, n$, as

$$
y_{k n}(x) \equiv \mu_{k n}^{-1} \sum_{j=1}^{n} w_{j n} y_{k j}^{(n)} K\left(x, x_{j n}\right) .
$$

It is natural to expect the difference between the two sides of (1), with $\mu_{k}$ and $y_{k}(x)$ replaced by $\mu_{k n}$ and $y_{k n}(x)$, respectively, to be expressible in terms of the error function (4). In fact,

$$
\mu_{k n} y_{k n}(x)-\int_{a}^{b} K(x, t) y_{k n}(t) d t=\mu_{k n}^{-1} \sum_{j=1}^{n} w_{j n} y_{k j}^{(n)} \eta_{n}\left(x, x_{j n}\right),
$$

where $\eta_{n}(x, t)$ is defined by (4).

Let, further, $\left\{y_{m}^{*}(x)\right\}, m=1, \ldots, r$, form an orthonormal base of all characteristic functions of $K(x, t)$ corresponding to $\mu_{k}$; then for every $n$ with $\mu_{k n} \neq 0$, there exist coefficients $c_{k m}^{(n)}, m=1, \ldots, r$, such that the error function 


$$
e_{k n}(x) \equiv y_{k n}(x)-\sum_{m=1}^{r} c_{k m}^{(n)} y_{m}^{*}(x)
$$

is of minimal norm. In fact,

$$
c_{k m}^{(n)}=\left(y_{k n}, y_{m}^{*}\right), \quad m=1, \ldots, r
$$

and, consequently,

(9) $\left(e_{k n}, y\right)=0$ for every characteristic function $y(x)$ of $K(x, t)$ corresponding to $\mu_{k}$.

The functions $e_{k n}(x)$ and $\tilde{y}_{k n}(x) \equiv y_{k n}(x)-e_{k n}(x)$ are called the error function and the characteristic function, respectively, associated with $y_{k n}(x)$. Now, if the approximate numerical solution $y_{k n}^{*}(x)$ for a characteristic function is taken to be of norm 1 , i.e., $y_{k n}^{*}(x)=\left\|y_{k n}\right\|^{-1} y_{k n}(x)$, it can be shown that the characteristic function $Y_{k n}(x)$ of norm 1 corresponding to $\mu_{k}$ such that the error function $e_{k n}^{*}(x) \equiv y_{k n}^{*}(x)-Y_{k n}(x)$ is of minimal norm, assumes the form

$$
Y_{k n}(x)= \begin{cases}R_{k n}^{-1} \tilde{y}_{k n}(x), & R_{k n} \neq 0, \\ y_{l}(x) \quad \text { with } \mu_{l}=\mu_{k}, & R_{k n}=0,\end{cases}
$$

where $R_{k n}=\left\|\tilde{y}_{k n}\right\|$. Also, since by (9) $\left\|y_{k n}\right\|^{2}=R_{k n}^{2}+\left\|e_{k n}\right\|^{2}$, we have

$$
e_{k n}^{*}(x)=\left\|y_{k n}\right\|^{-1}\left[e_{k n}(x)-\left(\left\|y_{k n}\right\|-R_{k n}\right) Y_{k n}(x)\right]
$$

$$
=\left\|y_{k n}\right\|^{-1}\left[e_{k n}(x)-\frac{\left\|e_{k n}\right\|^{2}}{\left\|y_{k n}\right\|+R_{k n}} Y_{k n}(x)\right] \text {. }
$$

4. Error Estimate and Convergence. For the sake of conciseness of presentation, the following definitions are introduced:

$\mu_{k}$ and $\mu_{k n}, k=1, \ldots, r$, are the $r$ largest (smallest) characteristic values

of $K(x, t)$ and the $r$ largest (smallest) eigenvalues of (2), respectively, such

that $\mu_{i} \geqslant \mu_{i+1}\left(\mu_{i} \leqslant \mu_{i+1}\right)$ and $\mu_{i n} \geqslant \mu_{i+1, n}\left(\mu_{i n} \leqslant \mu_{i+1, n}\right), i=1, \ldots$, $r-1$.

In the following, $F(x, t)$ is a kernel defined in $I \times I$.

$U(F)$ and $U_{n}(F)$ are the set of all characteristic values of $F(x, t)$ and the set of all eigenvalues of the matrix $F^{(n)}$ with $F_{i j}^{(n)} \equiv w_{j n} F\left(x_{i n}, x_{j n}\right), i, j=1$, $\ldots, n$, respectively.

$\lambda_{k}(F)$ and $\lambda_{k n}(F)$ are the $k$ th real elements of $U(F)$ and $U_{n}(F)$, respectively, in the ordering determined by that of the $\mu_{k}$ and the $\mu_{k n}$ in (11).

$M_{k}(F)$ and $M_{k n}(F), k=1, \ldots, r$, are the moduli of the $r$ elements of $U(F)$ and $U_{n}(F)$, respectively, of largest modulus, such that $M_{i}(F) \geqslant M_{i+1}(F)$ and $M_{i n}(F) \geqslant M_{i+1, n}(F), i=1, \ldots, r-1$.

$$
\begin{gathered}
Q(F, u) \equiv \int_{a}^{b} \int_{a}^{b} F(x, t) u(t) \overline{u(x)} d x d t, \\
Q_{n}(F, u) \equiv \sum_{i, j=1}^{n} w_{i n} w_{j n} F\left(x_{i n}, x_{j n}\right) u_{j} \bar{u}_{i},
\end{gathered}
$$




$$
\begin{gathered}
\delta_{n}(F, x, t) \equiv \sum_{i=1}^{n} w_{i n} F\left(x, x_{i n}\right) F\left(x_{i n}, t\right)-\int_{a}^{b} F(x, z) F(z, t) d z, \\
D_{n}(F, u) \equiv \int_{a}^{b} \int_{a}^{b} \delta_{n}(F, x, t) \overline{u(x)} u(t) d x d t, \\
D_{n}^{*}(F, u) \equiv \sum_{i, j=1}^{n} w_{i n} w_{j n} \delta\left(F, x_{i n}, x_{j n}\right) u_{j} \bar{u}_{i}, \\
A_{k n}(F) \equiv\left[\max \left\{\sum_{i=1}^{n} w_{i n}\left|\int_{a}^{b} \delta_{n}\left(F, x, x_{i n}\right) \overline{u(x)} d x\right|^{2} \mid u \in V_{k}(F)\right\}\right]^{1 / 2}, \\
B_{k n}(F) \equiv\left[\max \left\{\int_{a}^{b}\left|\sum_{i=1}^{n} w_{i n} \bar{u}_{i} \delta_{n}\left(F, x_{i n}, x\right)\right|^{2} d x \mid u \in V_{k n}(F)\right\}\right]^{1 / 2} ; \\
\alpha_{n} \equiv\left[\int_{a}^{b} \int_{a}^{b}\left|\eta_{n}(x, t)\right|^{2} d x d t\right]^{1 / 2}, \beta_{n} \equiv\left[\sum_{i, j=1}^{n} w_{i n} w_{j n}\left|\eta_{n}\left(x_{i n}, x_{j n}\right)\right|^{2}\right]^{1 / 2}, \\
\gamma_{n} \equiv\left[\sum_{i=1}^{n} w_{i n} \int_{a}^{b}\left|\eta_{n}\left(x, x_{i n}\right)\right|^{2} d x\right]^{1 / 2}, \\
\rho_{n} \equiv \max \left(\alpha_{n}, \beta_{n}\right),
\end{gathered}
$$

where $\eta_{n}(x, t)$ is defined by (4).

$y_{k n}(x)$ and $e_{k n}(x)$ are, respectively, the function (7) and the error function associated with it as defined in Section 3.

The new error estimates for the eigenvalues obtained in this paper are now summarized in the following two theorems:

THEOREM 1. If, with Definitions (11) and (24), $\nu_{k n} \equiv \max \left(\left|\mu_{k n}\right|,\left|\mu_{k}\right|\right) \geqslant C \sqrt{\rho_{n}}$ for some $C>1$, then

(a) $\left|\mu_{k n}-\mu_{k}\right| \leqslant \nu_{k n}^{-1}\left(\gamma_{n}+\rho_{n}\right)\left[1-\nu_{k n}^{-2} \rho_{n}\right]^{-1 / 2} \leqslant \nu_{k n}^{-1}\left(\gamma_{n}+\rho_{n}\right)\left[1-C^{-2}\right]^{-1 / 2}$,

$$
\left|\mu_{1 n}-\mu_{1}\right| \leqslant \gamma_{n}\left[\nu_{1 n}^{2}-\rho_{n}\right]^{-1 / 2}
$$

THEOREM 2. Let $\mu_{1 n}^{+} \geqslant \mu_{2 n}^{+} \geqslant \ldots \geqslant \mu_{r n}^{+}>0, \mu_{1 n}^{-} \leqslant \mu_{2 n}^{-} \leqslant \ldots \leqslant \mu_{s n}^{-}<0$, be the $r$ largest positive and the $s$ smallest negative eigenvalues of $(2)$, and let $\mu_{1}^{+} \geqslant$ $\mu_{2}^{+} \geqslant \ldots \geqslant \mu_{r}^{+}>0>\mu_{s}^{-} \geqslant \ldots \geqslant \mu_{2}^{-} \geqslant \mu_{1}^{-}$be the corresponding characteristic values of $K(x, t)$. 
If the integration rule $S$ is convergent with respect to $K(x, t)$ and satisfies (3), then

$$
\lim _{n \rightarrow \infty} \mu_{i n}^{+}=\mu_{i}^{+}, \quad \lim _{n \rightarrow \infty} \mu_{j n}^{-}=\mu_{j}^{-}, \quad i=1, \ldots, r, j=1, \ldots, s ;
$$

and the convergence is uniform in $i$ and $j$, so that for every $C>1$,

$$
\left|\mu_{i n}^{+}-\mu_{i}^{+}\right| \leqslant q_{n}, \quad\left|\mu_{j n}^{-}-\mu_{j}^{-}\right| \leqslant q_{n}, \quad i=1, \ldots, r, j=1, \ldots, s,
$$

$$
q_{n} \equiv \max \left\{C,\left[C^{2}-1\right]^{-1 / 2}\right\} \sqrt{\gamma_{n}+\rho_{n}}, \quad \text { where } \gamma_{n} \text { and } \rho_{n} \text { are defined by (24). }
$$

Theorem 2 is a generalization of Wielandt's results.

The error estimate for the approximate numerical solution of (1) is given by:

THEOREM 3. The error function defined by (25) satisfies (see Definitions (4), (11) and (12))

where

$$
\begin{aligned}
\left|e_{k n}(x)\right| \leqslant\left|\mu_{k}^{-1} \mu_{k n}^{-1}\right|\left\{\sum _ { j = 1 } ^ { n } w _ { j n } | y _ { k j } ^ { ( n ) } | \left[\max _{I}\left|\eta_{n}\left(x, x_{j n}\right)\right|\right.\right. & \left.+q_{k n} I_{j n} \sqrt{F(x)}\right] \\
& \left.+\left|\mu_{k n}-\mu_{k}\right| G_{n}(x)\right\}
\end{aligned}
$$

$$
\begin{gathered}
F(x) \equiv \int_{a}^{b}|K(x, t)|^{2} d t, \quad G_{n}(x) \equiv\left[F(x)+\eta_{n}(x, x)\right]^{1 / 2}, \\
q_{k n} \equiv \sup \left\{\left|\mu_{k n}-\lambda\right|^{-1} \mid \lambda \in U(K), \lambda \neq \mu_{k}\right\}, \\
I_{j n} \equiv\left[\int_{a}^{b}\left|\eta_{n}\left(x, x_{j n}\right)\right|^{2} d x\right]^{1 / 2} .
\end{gathered}
$$

This bound for $e_{k n}(x)$, and consequently that for the function $e_{k n}^{*}(x)$ defined by (10), are improvements, by a factor of $O\left(n^{-1 / 2}\right)$, of a similar error estimate obtained in [4].

Error estimates for the eigenvalues in special cases are given in Section 5.

An immediate consequence of Theorem 2, analogous to the one which follows from the convergence theorem in [1], is:

If the integration rule $S$ is convergent with respect to $K(x, t)$, then $e_{k n}(x)$ and $e_{k n}^{*}(x)$ converge to 0 uniformly in $I$.

Remark. If $K(x, t)$ satisfies a Lipschitz condition of the form $|K(u, t)-K(v, t)| \leqslant$ $L|u-v|^{p}, 0<p \leqslant 1$, in $I \times I$, then (see Definition (4))

$$
\begin{aligned}
\left|e_{k n}(x)\right| \leqslant\left|\mu_{k}^{-1}\right|\left\{\left|\mu_{k n}^{-1}\right| \sum_{j=1}^{n} w_{j n}\left|y_{k j}^{(n)}\right|\left[\max _{I}\left|\eta_{n}\left(x, x_{j n}\right)\right|+q_{k n} I_{j n} \sqrt{F(x)}\right]\right. \\
\left.+\left|\mu_{k n}-\mu_{k}\right|\left[\max _{m}\left|y_{k m}^{(n)}\right|+\frac{2^{-p} L}{\left|\mu_{k n}\right|} \max _{0<m<n}\left(x_{m+1, n}-x_{m n}\right)^{p} \sum_{j=1}^{n} w_{j n}\left|y_{k j}^{(n)}\right|\right]\right\}
\end{aligned}
$$

where $x_{0 n}=a$ and $x_{n+1, n}=b$.

Since the estimate for $e_{k n}(x)$ involves an estimate for $q_{k n}$, it can be found only if the multiplicity of $\mu_{k}$ is known. Such an estimate is obtainable, for instance, when $\mu_{k}$ is a simple characteristic value and in this case we deduce by Theorem 2, Eq. (38) of [6] and Lemma 1 in Section 6: 
COROLlARY 1. If $\mu_{k}$ is a simple characteristic value of $K(x, t)$ and the integration rule is convergent with respect to $K(x, t)$ and satisfies (3), then for some choice of eigenvectors $y_{k}^{(n)}$ such that $\left|y_{k}^{(n)}\right|_{n}=1$ (see Definitions (5) and (7)), $y_{k n}(x) \rightarrow y_{k}(x)$ uniformly in $I$, and so does also $\left\|y_{k n}\right\|^{-1} y_{k n}(x)$.

By (7) it follows that

where

$$
\left\|y_{k n}\right\|^{2}=\mu_{k n}^{-2} \sum_{i, j=1}^{n} w_{i n} w_{j n} \overline{y_{k i}^{(n)}} y_{k j}^{(n)} G\left(x_{i n}, x_{j n}\right)
$$

$$
G(\dot{x}, t) \equiv \int_{a}^{b} \overline{K(z, x)} K(z, t) d z=\int_{a}^{b} K(x, z) K(z, t) d z
$$

In the case where $G(x, t)$ cannot be determined exactly, an approximation $c_{k n}$ of $\left\|y_{k n}\right\|$ is found by applying some quadrature formula for determining $G(x, t)$ at the points $\left(x_{i n}, x_{j n}\right)$; the approximate solution for a characteristic function is then taken to be $c_{k n}^{-1} y_{k n}(x)$, and the error estimate is

$$
\begin{aligned}
\tilde{e}_{k n}(x) & =\left|c_{k n}^{-1} y_{k n}(x)-R_{k n}^{-1} \tilde{y}_{k n}(x)\right| \leqslant\left|\left(c_{k n}^{-1}-\left\|y_{k n}\right\|^{-1}\right) y_{k n}(x)\right|+\left|e_{k n}^{*}(x)\right| \\
& =\left(c_{k n}\left\|y_{k n}\right\|\right)^{-1}\left|\left(c_{k n}-\left\|y_{k n}\right\|\right) y_{k n}(x)\right|+\left|e_{k n}^{*}(x)\right|
\end{aligned}
$$

where $e_{k n}^{*}(x)$ is given by $(10)$.

5. Improved Error Estimates for Simple Characteristic Values and for Positive-

Definite Kernels. An error estimate for a simple characteristic value can be improved if the approximate eigenvalue $\mu_{k n}$ satisfies the inequality (see Definition (11))

$$
\left|\mu_{k n}-\mu_{k}\right|<\min _{i \neq k}\left|\mu_{k n}-\mu_{i}\right| .
$$

If the integration rule $S$ is convergent with respect to $K(x, t)$ and satisfies (3), and $\mu_{k}$ is a simple characteristic value, then by Theorem 2 there exists an integer $N$ such that the above inequality holds for $n>N$, and by Lemma 5 (stated in Section 6)

$$
\left|\mu_{k n}-\mu_{k}\right|=\inf \left\{\left|\mu_{k n}-\lambda\right| \mid \lambda \in U(K)\right\} \leqslant\left|\mu_{k n}^{-1}\right|\left\|y_{k n}\right\|^{-1} \gamma_{n} .
$$

An error estimate for a positive-definite kernel is obtained from the following theorem:

THEOREM 4. Let $\widetilde{\mu}_{k n} \in U_{n}(K)$ and $\widetilde{\mu}_{k} \in U(K), k=1, \ldots, n$, such that $\left|\tilde{\mu}_{k n}\right|=M_{k n}(K)$ and $\left|\tilde{\mu}_{k}\right|=M_{k}(K)$ (see Definitions (12), (14) and (4)). Then

$$
\tilde{\mu}_{k n}^{2}-\tilde{\mu}_{k}^{2} \mid \leqslant M_{1}\left(\eta_{n}\right)+M_{1 n}\left(\eta_{n}\right), \quad k=1,2, \ldots,
$$

where $\tilde{\mu}_{k n}=0$ for $k>n$.

Corollary 2. If $K(x, t)$ is positive-definite and (see Definitions (11) and (12)), $\mu_{k n}>-\min \left\{\lambda \mid \lambda \in U_{n}(K)\right\}$, then, $\left|\mu_{k n}^{2}-\mu_{k}^{2}\right| \leqslant M_{1}\left(\eta_{n}\right)+M_{1 n}\left(\eta_{n}\right)$.

We also obtain

CoROllaRY 3. $\left|\mu_{n+k}\right| \leqslant \sqrt{M_{1}\left(\eta_{n}\right)}, k=1,2, \ldots$.

6. Discussion of the Theorems. To obtain the final results presented in Theorems $1-4$, the following lemmas are necessary using the definitions introduced at the beginning of Section 4: 
LEMMA 1. Let $u_{n}=\left(u_{n 1}, \ldots, u_{n n}\right)$ and $A_{n}=\left(a_{i j}^{(n)}\right)$ be, respectively, sequences of vectors and $n \times n$ matrices with complex elements. Then (see Definition (5))

(a) $\left|u_{n}\right|_{n}^{2}=\sum_{k=1}^{n}\left|\left(u_{n}, z_{k}\right)_{n}\right|^{2}=\sum_{k=1}^{n}\left|\left(z_{k}, u_{n}\right)_{n}\right|^{2}$ for every sequence $z_{k}, k=$ $1, \ldots, n$, satisfying

$$
\left(z_{p}, z_{q}\right)_{n}=\delta_{p q}, \quad p, q=1, \ldots, n .
$$

(b) If the integration rule $S$ satisfies (3), then

$$
\begin{aligned}
\lim _{n \rightarrow \infty} \max _{i}\left|u_{n i}\right|=0 \text { implies } \lim _{n \rightarrow \infty} \sum_{i=1}^{n} w_{i n} u_{n i}=\lim _{n \rightarrow \infty} \sum_{i=1}^{n} w_{i n}\left|y_{k i}^{(n)} u_{n i}\right| & =0, \\
k & =1,2, \ldots,
\end{aligned}
$$

and

$$
\lim _{n \rightarrow \infty} \max _{i, j}\left|a_{i j}^{(n)}\right|=0 \text { implies } \lim _{n \rightarrow \infty} \sum_{i, j=1}^{n} w_{i n} w_{j n} a_{i j}^{(n)}=0 .
$$

LEMMA 2. If $\lambda_{k} \equiv \lambda_{k}(F)=\lambda_{1}(F)$, where $F(x, t)$ is a Hermitian kernel defined in $I \times I$, then (see Definitions (13), (22), (17) and (20))

$$
\lambda_{k}\left(\lambda_{k}-\lambda_{k n}(F)\right) \leqslant A_{k n}(F)\left[1-\lambda_{k}^{-2} \max _{V_{k}(F)}\left|D_{n}(F, u)\right|\right]^{-1 / 2} .
$$

LEMMA 3. If $\lambda_{k n} \equiv \lambda_{k n}(F)=\lambda_{1 n}(F)$, where $F(x, t)$ is a Hermitian kernel defined in $I \times I$, then (see Definitions (13), (23), (18) and (21))

$$
\lambda_{k n}\left(\lambda_{k n}-\lambda_{k}(F)\right) \leqslant B_{k n}(F)\left[1-\lambda_{k n}^{-2} \max _{V_{k n}(F)}\left|D_{n}^{*}(F, u)\right|\right]^{-1 / 2} .
$$

This lemma is a consequence of Weyl's theorem [8, p. 445]:

Lemma 4. Let $D(x, t) \equiv F(x, t)-G(x, t)$, where $F(x, t)$ and $G(x, t)$ are Hermitian kernels defined in $I \times I$; then (see Definitions (13), (15) and (16))

(a) if $Q(D, u) \geqslant 0$ for every $u(x)$, then $\lambda_{k}(F) \geqslant \lambda_{k}(G), k=1,2, \ldots$;

(b) if $Q_{n}(D, u) \geqslant 0$ for every $u \in C_{n}$, then $\lambda_{k n}(F) \geqslant \lambda_{k n}(G), k=1, \ldots, n$.

The next and last lemma is used to obtain the improved error estimates for simple characteristic values mentioned in Section 5.

Lemma 5. With Definitions (11), (12), (7) and (24),

$$
D_{k n} \equiv \inf \left\{\left|\mu_{k n}-\lambda\right| \mid \lambda \in U(K)\right\} \leqslant\left|\mu_{k n}^{-1}\right|\left\|y_{k n}\right\|^{-1} \gamma_{n} .
$$

This is a slight improvement of the result obtained in [3].

The proofs of Lemmas 1, 4 and 5 are straightforward ([6, Lemmas 1,5 and 2, respectively]), whereas those of Lemmas 2 and 3 require some special devices ([6, Lemmas 3 and 4 , respectively]).

The first four lemmas are used to establish part (a) of Theorem 1 by the following steps:

1. Application of Lemma 2 and part (b) of Lemma 4 to obtain (see Definitions (11), (22), (17) and (20))

$$
\mu_{k}\left(\mu_{k}-\mu_{k n}\right) \leqslant A_{k n}(L)\left[1-\mu_{k}^{-2} \max _{V_{k}(L)}\left|D_{n}(L, u)\right|\right]^{-1 / 2},
$$

where

$$
L(x, t) \equiv K(x, t)-\sum_{p=1}^{k-1}\left(\mu_{p}-\mu_{k}\right) y_{p}(x) \overline{y_{p}(t)}
$$


2. Application of Lemma 3 and part (a) of Lemma 4 to obtain (see Definitions (11), (23), (18) and (21))

$$
\mu_{k n}\left(\mu_{k n}-\mu_{k}\right) \leqslant B_{k n}\left(L_{n}\right)\left[1-\mu_{k n}^{-2} \max _{V_{k n}\left(L_{n}\right)}\left|D_{n}^{*}\left(L_{n}, u\right)\right|\right]^{-1 / 2},
$$

where

$$
L_{n}(x, t) \equiv K(x, t)-\sum_{p=1}^{k-1}\left(\mu_{p n}-\mu_{k n}\right) y_{p n}(x) \overline{y_{p n}(t)} .
$$

3. Bounding of $A_{k n}(L), \max \left\{\left|D_{n}(L, u)\right| \mid u \in V_{k}(L)\right\}, B_{k n}\left(L_{n}\right)$ and $\max \left\{\left|D_{n}^{*}\left(L_{n}, u\right)\right| \mid u \in V_{k n}\left(L_{n}\right)\right\}$ in terms of $\gamma_{n}$ and $\rho_{n}$ defined by (24), which is a matter of pure manipulations.

Theorem 2 follows from Lemma 1 and Theorem 1.

Theorem 3 is a consequence of (9) and the Parseval equality (equation of closedness $[5, \mathrm{p} .10])$ for the function $e_{k n}(x)$.

For the full proof of the above theorems the reader is referred to [6].

Finally, we come to the proof of Theorem 4, which terminates our discussion.

Proof of Theorem 4. The degenerate kernel

$$
G_{n}(x, t) \equiv \sum_{i=1}^{n} w_{i n} K\left(x, x_{i n}\right) K\left(x_{i n}, t\right)=\sum_{i=1}^{n} w_{i n} \overline{K\left(x_{i n}, x\right)} K\left(x_{i n}, t\right),
$$

is Hermitian and $G_{n}(x, t)=G(x, t)+\eta_{n}(x, t)$, where

$$
G(x, t) \equiv \int_{a}^{b} K(x, z) K(z, t) d z,
$$

therefore the characteristic values $\nu_{k n}$ of $G_{n}(x, t)$, where $\nu_{1 n} \geqslant \nu_{2 n} \geqslant \ldots \geqslant \nu_{n n} \geqslant$ $\nu_{n+1, n}=\ldots=0$, are related to those of $G(x, t)$, which are $\widetilde{\mu}_{k}^{2}$, by the inequalities $[8$, p. 445]:

$$
\left|\nu_{k n}-\tilde{\mu}_{k}^{2}\right| \leqslant M_{1}\left(\eta_{n}\right), \quad k=1,2, \ldots .
$$

The $\nu_{k n}, k=1, \ldots, n$, are exactly the eigenvalues of the matrix $L_{n} \equiv$ $\left(w_{i n} G\left(x_{i n}, x_{j n}\right)\right)$, which is similar to the Hermitian matrix $A^{(n)}$ defined by

$$
A_{i j}^{(n)} \equiv \sqrt{w_{i n} w_{j n}} G\left(x_{i n}, x_{j n}\right)=\sqrt{w_{i n} w_{j n}}\left[G_{n}\left(x_{i n}, x_{j n}\right)-\eta_{n}\left(x_{i n}, x_{j n}\right)\right] .
$$

Now, a procedure similar to that described in [8] for characteristic values of kernels leads to the inequalities

$$
\left|\tilde{\mu}_{k n}^{2}-v_{k n}\right| \leqslant M_{1 n}\left(\eta_{n}\right), \quad k=1,2, \ldots,
$$

where $\tilde{\mu}_{k n}=0$ for $k>n$, which together with (33) yields

$$
\tilde{\mu}_{k n}^{2}-\tilde{\mu}_{k}^{2} \mid \leqslant M_{1}\left(\eta_{n}\right)+M_{1 n}\left(\eta_{n}\right), \quad k=1,2, \ldots
$$

Corollary 3 follows from (33).

Acknowledgments. The author is indebted to I. Navot, Faculty of Electrical Engineering, Technion, I.I.T., and to J. Steinberg, Department of Mathematics, Technion, I.I.T., for their care in reading the manuscript and their comments. 
1. K. E. ATKINSON, "The numerical solution of the eigenvalue problem for compact integral operators," Trans. Amer. Math. Soc., v. 129, 1967, pp. 458-465. MR 36 \#3172.

2. C. T. H. BAKER, "The deferred approach to the limit for eigenvalues of integral equations," SIAM J. Numer. Anal., v. 8, 1971, pp. 1-10. MR 48 \#3276.

3. H. BRAKHAGE, "Zur Fehlerabshätzung für die numerische Eigenwertbestimmung bei Integralgleichungen," Numer. Math., v. 3, 1961, pp. 174-179. MR 23 \#A2185.

4. P. LINZ, "On the numerical computation of eigenvalues and eigenvectors of symmetric integral equations," Math. Comp., v. 24, 1970, pp. 905-910. MR 43 \#1461.

5. S. C. MIHLIN, Lectures on Linear Integral Equations, Fizmatgiz, Moscow, 1959; English transl., Russian Monographs and Texts on Advanced Math. and Phys., vol. 2, Gordon and Breach, New York; Hindustan, Delhi, 1960. MR 23 \#A490; 24 \#A3483.

6. E. RAKOTCH, Numerical Solution for Eigenvalues and Eigenfunctions of a Hermitian Ker. nel and an Error Estimate, Technical Report No. 38, Department of Computer Science, Technion, I.I.T, November 1974.

7. J. TODD, Introduction to the Constructive Theory of Functions, Academic Press, New York, 1963. MR 27 \#6061.

8. H. WEYL, "Das asymptotische Verteilungsgesetz der Eigenwerte linearer partieller Differentialgleichungen (mit einer anwendung auf die Theorie der Hohlraumstrahlung)," Math. Ann., v. 71, 1912, pp. 441-479.

9. H. WIELANDT, Error Bounds for Eigenvalues of Symmetric Integral Equations, Proc.

Sympos. Appl. Math., vol. 6, Amer. Math. Soc., Providence, R. I., 1956, pp. 261-282. MR $19,179$. 